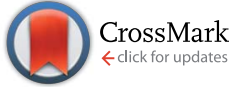

Cite this: RSC Adv., 2017, 7, 11880

Received 2nd December 2016 Accepted 23rd January 2017

DOI: $10.1039 / c 6 r a 27669 e$

rsc.li/rsc-advances

\section{3-93 bioactive glass/alginate composite scaffolds 3D printed under mild conditions for bone regeneration}

\author{
Guilin Luo, $\uparrow^{\mathrm{a}}$ Yufei Ma,,$^{\mathrm{ab}} \mathrm{Xu}$ Cui, ${ }^{\mathrm{a}}$ Lixin Jiang, ${ }^{\mathrm{a}}$ Mingming $\mathrm{Wu},{ }^{\mathrm{a}}$ Yang Hu, ${ }^{\text {ac }}$ \\ Yanfeng Luo, ${ }^{\text {' Haobo Pan }}{ }^{a}$ and Changshun Ruan*a
}

Composite scaffolds of type 13-93 bioactive glass (13-93 BG) and sodium alginate (SA), denoted 13-93 BG/ SA, in mass ratios of $0: 4,1: 4,2: 4$ and $4: 4$ were prepared for bone regeneration by $3 \mathrm{D}$ printing under mild conditions. All obtained scaffolds were completely interconnected. As the 13-93 BG/SA mass ratio increased from $0: 4$ to $4: 4$, the pore size improved from $340 \pm 27$ to $530 \pm 27 \mu \mathrm{m}$, porosity increased from $65.5 \pm 5.3$ to $87.5 \pm 3.9 \%$ and shrinkage ratios decreased by half. Moreover, 13-93 BG incorporation improved the compressive strength and modulus of the SA scaffold, achieving highest values of $16.74 \pm 1.78 \mathrm{MPa}$ and $79.49 \pm 7.38 \mathrm{MPa}$, respectively, in 13-93 BG/SA with a $2: 4$ mass ratio. Furthermore, in vitro apatite mineralization, the release of bioactive ions $\left(\mathrm{Mg}^{2+}\right.$ and $\left.\mathrm{SiO}_{4}{ }^{4-}\right)$ and a weakly alkaline $\mathrm{pH}$ were exhibited by scaffolds containing 13-93 BG, which further promoted the attachment, proliferation and osteogenic differentiation of rBMSCs on composite scaffolds. As a result, 3D-printed 13-93 BG/SA scaffold was an ideal candidate for bone regeneration.

\section{Introduction}

A new generation of tissue engineering scaffolds with good interconnection, biodegradability, bioactivity and adequate mechanical support is required for bone regeneration., ${ }^{1,2}$ Tissue engineering scaffolds have many essential features, among which composition and macro/nanoporous connected morphology are thought be critical in influencing cellular response. Biodegradable organicinorganic composite scaffolds have recently attracted attention as bone tissue mimics. ${ }^{3-6}$ Their main advantage is that altering the organic/inorganic material composition or ratio can change the properties of the resultant composite scaffold to satisfy the requirements of bone regeneration. ${ }^{5}$ Organic materials used for bone tissue engineering applications are usually natural polymers (such as collagen, chitosan and alginate) or synthetic polymers (such as polylactic acid (PLA) and polycaprolactone (PCL)), because of their biocompatibility and biodegradation. ${ }^{7,8}$ Inorganic materials used are usually hydroxyapatite (HAp), $\beta$-tricalcium phosphate $\left(\beta\right.$-TCP) and bioactive glasses because of their osteoconductivity. ${ }^{9-12}$

${ }^{a}$ Center for Human Tissue and Organs Degeneration, Institute Biomedical and Biotechnology, Shenzhen Institutes of Advanced Technology, Chinese Academy of Sciences, Shenzhen 518055, China. E-mail: cs.ruan@siat.ac.cn; Tel: +86 755 86585250

${ }^{b}$ Key Laboratory of Biorheological Science and Technology, Ministry of Education, Research Center of Bioinspired Materials Science and Engineering, College of Bioengineering, Chongqing University, Chongqing 400030, China

${ }^{c}$ Fiber and Biopolymer Research Institute, Department of Plant and Soil Science, Texas Tech University, Lubbock, TX 79403, USA

$\dagger$ These authors have contributed equally to this work.
The macro/nanoporous connected morphology of composite scaffolds has been investigated using various methods, most notably solvent casting, particulate leaching and freezedrying. ${ }^{\text {13-16 }}$ However, these conventional methods have many limitations. ${ }^{\mathbf{1 6}}$ For example, the pore size, morphology and distribution, and the external scaffold geometry, are difficult to control. Moreover, the application of non-physiological conditions prevents incorporation of biological components during the scaffold fabrication process. In the last decade, the development of 3D printing technology has allowed the fabrication of scaffolds with controlled and regular pore sizes, shapes and interconnectivity using computer aided design and computer aided manufacturing (CAM), suggesting that 3D printing could be used to construct individual scaffolds for bone regeneration., ${ }^{\mathbf{4 7 - 2 0}}$ Furthermore, composite scaffolds for tissue engineering have been fabricated by 3D printing under mild conditions (room or physiological temperature and organic-free solvent), which should be beneficial for designing multifunctional scaffolds containing bioactive proteins or living cells for medical therapy. ${ }^{\mathbf{4 , 5}, 17}$

Based on this background, biodegradable organic-inorganic composite scaffolds for bone regeneration were fabricated in this present study using 3D printing under mild conditions. Biocompatible and biodegradable alginate has been widely used in tissue engineering, growth factor and cell delivery, ${ }^{\mathbf{1 2 2 0 - 2 3}}$ and was selected as the organic component in this study. It is also a suitable biomaterial for preparing functional hydrogels, due to its water-solubility and cross-linking with ionic response. Furthermore, the sol-gel transition of alginate induced by 
multivalent cations, such as $\mathrm{Ca}^{2+}$, under mild conditions has been successfully developed as a novel stabilization method for 3D printing, which would be convenient for encapsulating bioactive proteins or cells in 3D printed scaffolds. ${ }^{24}$ Type 13-93 bioactive glass (13-93 BG) was used as the inorganic component to fabricate the composite scaffolds for bone repair. 13-93 BG is modified from the $45 \mathrm{~S} 5$ composition, but contains a higher $\mathrm{SiO}_{2}$ content and additional network modifiers, such as $\mathrm{K}_{2} \mathrm{O}$ and $\mathrm{MgO}$. It has been stated that 13-93 BG is an ideal biomaterial for bone regeneration, because it has the same osteoconductivity as 45S5 BG, but a higher mechanical strength and faster biodegradation ratio. ${ }^{25,26}$ However, the appropriate conditions for fabricating 13-93 BG scaffolds with controlled morphology and suitable mechanical properties for bone regeneration require investigation. Rahaman and collaborators successfully prepared 13-93 BG scaffolds with high strength and porosity for bone regeneration by slip casting, freeze extrusion fabrication and unidirectional freezing of camphene-based suspensions, and further testified the excellent performance of 13-93 BG in bone repair. ${ }^{27-29}$ However, BG scaffold preparing methods are inconvenient, requiring other organic solvents, additional sintering procedures, or cool temperatures. Furthermore, the consistency and repeatability of the pore architectures in 13-93 BG scaffolds prepared by these methods needs improving. Therefore, taking advantage of the sol-gel transition of alginate induced by multivalent cations to stabilize 3D printing could be an alternative method for fabricating 13-93 BG/alginate composite scaffolds under mild conditions.

These organic-inorganic composite scaffold fabricated under mild conditions combined both the characteristics of 13$93 \mathrm{BG}$ and alginate with the advantage of 3D printing to control pore size and individual architecture. 13-93 BG/alginate composite scaffolds were fabricated with mass ratios of $0: 4$ (0\%), $1: 4(20 \%), 2: 4(33.3 \%)$ and $4: 4(50 \%)$ using 3D printing under mild conditions with the same geometry and plotting parameters. Their structures, mechanical properties, in vitro apatite mineralization abilities and cell response to rat bone mesenchymal stem cells (rBMSCs) were further investigated.

\section{Materials and methods}

\section{Synthesis and characterization of 13-93 BG powders}

According to a previous study, ${ }^{27} 13-93 \mathrm{BG}$ was prepared by melting the required quantities of $\mathrm{SiO}_{2}, \mathrm{CaCO}_{3}, \mathrm{Na}_{2} \mathrm{CO}_{3}, \mathrm{~K}_{2} \mathrm{CO}_{3}, \mathrm{MgCO}_{3}$ and $\mathrm{NaH}_{2} \mathrm{PO}_{4}$ (analytical grade, Sinopharm Chemical Reagent Co., Ltd., China) in a platinum/rhodium crucible in air for $2 \mathrm{~h}$ at $1300^{\circ} \mathrm{C}$. The melt was stirred twice at the isothermal temperature and quenched between two steel plates. Each glass powder was crushed with a hardened steel mortar and pestle, and then sieved through a stainless-steel sieve to give particle sizes of $<40 \mu \mathrm{m}$. The 13-93 BG composition (in mol\%) was $6 \mathrm{Na}_{2} \mathrm{O} \cdot 8 \mathrm{~K}_{2} \mathrm{O} \cdot 8 \mathrm{MgO} \cdot 22 \mathrm{CaO} \cdot 54 \mathrm{SiO}_{2}-$ $\cdot 2 \mathrm{P}_{2} \mathrm{O}_{5}$. X-ray diffraction (XRD, Bruker, Germany) and Fourier translation infrared spectroscopy (FTIR, Spectrum 2000, PerkinElmer, USA) were used to analyze the 13-93 BG powders, and their morphologies were characterized by scanning electron microscopy (SEM, Jeol JSM6510, Tokyo, Japan).

\section{Fabrication of 13-93 BG/SA scaffolds by 3D printing}

The scaffolds were 3D printed as shown in Fig. 1. Sodium alginate powders (SA, Mingyue Chemical Reagent, China) and 13-93 BG powders were mixed until homogeneous using 13-93 BG/SA mass ratios of $0: 4(0 \% 13-93$ BG), $1: 4(20 \%), 2: 4$ (33.3\%) and $4: 4(50 \%)$. Printable inks were then prepared by mixing $1 \mathrm{~g}$ of 13-93 BG/SA with $5 \mathrm{~g}$ of polyvinyl alcohol (PVA) in a $6 \mathrm{wt} \%$ solution (Sigma-Aldrich, $M_{\mathrm{w}}=13000-23000$ ). After stirring until homogeneous, the inks were loaded into a printing tube and the scaffolds were printed using a printing needle (406 $\mu \mathrm{m}$ diameter) on a Bioscaffolder 2.1 platform (GeSiM, Germany) with computer-assisted design (Fig. 1a). The composite inks were extruded at room temperature using dosing pressures ranging from 400 to $500 \mathrm{kPa}$ at a constant dispensing speed of $25 \mathrm{~mm} \mathrm{~s}^{-1}$. The preformed scaffolds (Fig. 1b) were soaked in $1 \mathrm{M} \mathrm{CaCl}_{2}$ aqueous solution for $5 \mathrm{~h}$ to achieve crosslinking, and then washed with deionized water three times. Finally, the scaffolds were lyophilized for use in subsequent experiments (Fig. 1c).

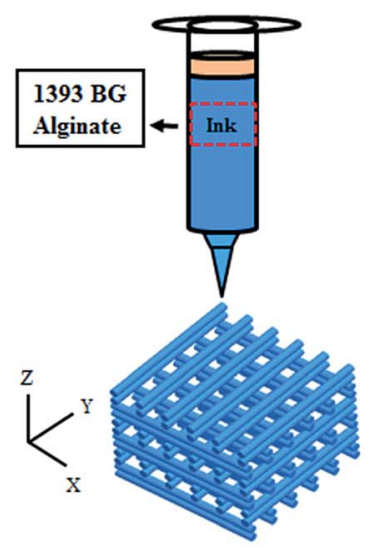

a) $3 D$ printing

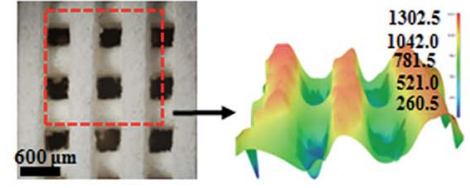

c) scaffolds in dry state (3d image)

$1 \mathrm{M} \mathrm{CaCl}_{2} 5 \mathrm{~h}$;

lyophilized;

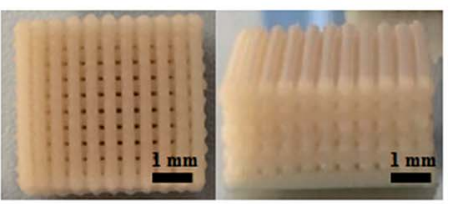

b) scaffolds in wet state (optical images)

Fig. 1 Fabrication process for BG/SA composite scaffolds: (a) computer assisted design model and bio-inks; (b) optical images of scaffolds in a wet state; and (c) 3D micrographs of scaffolds in a dry state. (Print model: XXYY pattern, $406 \mu \mathrm{m}, 400-500 \mathrm{kPa}$ at a speed of $25 \mathrm{~mm} \mathrm{~s}{ }^{-1}$ ). 


\section{Characterization of scaffold morphology, porosity and shrinkage}

The morphology and pore sizes of the 3D-printed 13-93 BG/SA scaffolds were characterized by SEM.

The scaffold porosity $(\rho)$ was measured using a liquid replacement method. ${ }^{4,30}$ In brief, a scaffold was placed in a graduated cylinder containing dimethyl formamide (DMF) of a recorded volume $\left(\nu_{1}\right)$. The cylinder was then placed under vacuum to force DMF into the scaffold pores. When no more bubbles appeared, the final volume was recorded as $\nu_{2}$. Finally, the scaffold was removed and the volume of remaining DMF was recorded as $\nu_{3}$. The porosity, $\rho$, of the scaffold was calculated according to eqn (1):

$$
\rho=\frac{v_{1}-v_{3}}{v_{2}-v_{3}} \times 100 \%
$$

Shrinkage was calculated by measuring the scaffold length and height before and after drying. The values of $\rho$ and shrinkage were averages from five samples.

\section{Mechanical properties of 13-93 BG/SA scaffolds}

The compressive strengths of the 13-93 BG/SA scaffolds $(6 \times 6 \times$ $6 \mathrm{~mm}$ ) were measured using an electromechanical universal testing machine (CMT6000, China) at a crosshead speed of 0.5 $\mathrm{mm} \mathrm{min}^{-1}$. A compressive deformation of $70 \%$ was achieved for all scaffolds at room temperature. The experiment was repeated using five samples.

\section{In vitro apatite mineralization and ionic release of 13-93 BG/SA scaffolds}

The apatite mineralization abilities of the 13-93 BG/SA scaffolds were investigated by soaking scaffolds in simulated body fluid (SBF), using $200 \mathrm{~mL} \mathrm{~g}^{-1}$ of scaffold mass, at $37^{\circ} \mathrm{C}$ for 0 and 10 days. Three samples were used as replicates. The morphologies and elemental compositions of the scaffold apatite mineralization layers were measured by SEM and energy-dispersive spectroscopy (EDS).

Ions released by the 13-93 BG/SA scaffolds were studied by soaking the scaffolds in $1.5 \mathrm{~mL}$ PBS at $37{ }^{\circ} \mathrm{C}$ for $1,3,5$ and 7 days. At predetermined time points, $1 \mathrm{~mL}$ aliquots of the release solution were taken and centrifuged at $10000 \mathrm{rpm}$ for $3 \mathrm{~min}$, with $1 \mathrm{~mL}$ of fresh PBS solution added to the scaffolds for further incubation. The solution $\mathrm{pH}$ and the release of calcium, magnesium and silicate ions were measured using a pH meter and inductively coupled plasma atomic emission spectroscopy (ICP-OES, Optima 7100 DV, PerkinElmer Co. USA), respectively.

\section{Seeding and cultivation of rBMSCs on scaffolds}

Primary rat bone mesenchymal stem cells (rBMSCs) were obtained from femurs and tibiae of Sprague-Dawley (SD) rats (140$160 \mathrm{~g}$ ) according to a known method, ${ }^{31}$ and cells at passage 8 were used for experiments. The cell experiment protocols were approved by Shenzhen Institute of Advanced Technology, Chinese Academy of Science. All experiments were performed in compliance with the Regulations for the Administration of Affairs Concerning Experimental Animals in China.

rBMSCs were cultivated in Dulbecco's modified Eagle's medium (DMEM, low glucose) containing 10\% fetal calf serum, $10 \mathrm{U} \mathrm{mL}^{-1}$ penicillin and $100 \mu \mathrm{g} \mathrm{mL}{ }^{-1}$ streptomycin at $37{ }^{\circ} \mathrm{C}$ under $5 \% \mathrm{CO}_{2}$ in air. Before cell seeding, the cell culture scaffolds $\left(5 \times 5 \times 1.56 \mathrm{~mm}^{3}\right)$ were immersed in $75 \%$ ethanol for $6 \mathrm{~h}$, washed three times with PBS and then immersed in culture medium without fetal calf serum for $12 \mathrm{~h}$. After removing the culture medium, the scaffolds were transferred to 24 -well plates and $2 \times 10^{4}$ cells were seeded onto each sample. The medium was renewed every 2 days.

\section{Viability and morphology of rBMSCs on scaffolds}

After cell culture for 1, 3 and 5 days, the proliferation of rBMSCs seeded on the scaffolds was evaluated using cell counting kit-8 (CCK-8, Dojindo). ${ }^{32}$ Absorbance was measured at $450 \mathrm{~nm}$ using a microplate reader. All experiments were performed in triplicate.

Morphologies of rBMSCs on the scaffolds were determined by fluorescence microscopy (Olympus, Tokyo) after $36 \mathrm{~h}$ of culture. In this experiment, the rBMSCs were stained with $2 \mu \mathrm{g} \mathrm{mL}$ fluorescent dye (5/6-( $\mathrm{N}$-succinimidyloxycarbonyl)-3' , $^{\prime}$-O,O-diacetyl fluorescein) before use. ${ }^{33}$

\section{Alkaline phosphatase (ALP) activity assay}

rBMSCs were seeded on the scaffolds under culture conditions in growth medium (DMEM, 10\% FBS). ALP activity was measured after 7 and 14 days of culture, as previously described. ${ }^{2}$ Briefly, the cells were washed three times with PBS and disrupted at $4{ }^{\circ} \mathrm{C}$ for $30 \mathrm{~min}$ using cell lysis buffer. The cell lysis buffer was then collected by centrifugation and stored at $-20{ }^{\circ} \mathrm{C}$. The ALP activity of the cell lysis buffer was determined using an alkaline phosphatase kit (Beyotime Institute of Biotechnology, Shanghai, China). The total protein content of the cell lysis buffer was determined using a BCA protein assay kit (Pierce Chemical Co., Rockford, USA), read at $562 \mathrm{~nm}$ and calculated according to a series of bovine serum albumin (BSA) standards. ALP activity was expressed as absorbance at $405 \mathrm{~nm}$ (OD value) per milligram of total cellular proteins. Cell culture plates were used as the control. All experiments were performed in triplicate.

\section{Quantitative real-time polymerase chain reaction (qRT-PCR)}

The osteogenic differentiation of rBMSCs seeded on scaffolds ( $0: 4$ and $2: 4$ groups) was further assessed by qRT-PCR after coculture for 7 and 14 days. The relative mRNA expression levels of commonly used bone markers, including osteopontin (OPN), osteocalcin (OCN), type I collagen (Col-I), and alkaline phosphatase (ALP) (primer pairs used are shown in Table 2), were measured. Total RNA isolation for qRT-PCR samples was conducted using an RNAsimple Total RNA Kit (Tiangen, Beijing, China). Complementary DNA (cDNA) was then reversetranscribed from $1 \mu \mathrm{g}$ of total RNA using a RevertAid First Strand cDNA Synthesis Kit (ThermoFisher Scientific, Massachusetts, USA) according to manufacturer instructions. Realtime PCR was performed on SYBR Green PCR Master Mix 
(Toyobo Life Science, Osaka, Japan) and the reaction was carried out using an ABI 7500 system (Applied Biosystems, Massachusetts, USA). Finally, housekeeping gene glyceraldehyde-3phosphate dehydrogenase (GAPDH) was used to normalize the relative mRNA expression level of each gene and quantification was based on the cycle threshold (CT) values.

\section{Statistical analysis}

For all experiments, the data were expressed as mean \pm standard deviation (SD). One-way ANOVA was performed to analyze variables, with $p$-values $<0.05$ considered statistically significant differences.

\section{Results}

\section{Characterization of 13-93 BG powders}

The 13-93 BG powders $\left(6 \mathrm{Na}_{2} \mathrm{O} \cdot 8 \mathrm{~K}_{2} \mathrm{O} \cdot 8 \mathrm{MgO} \cdot 22 \mathrm{CaO} \cdot 54 \mathrm{SiO}_{2}\right.$ $\cdot 2 \mathrm{P}_{2} \mathrm{O}_{5}$ ) were successfully prepared using appropriate quantities of the raw materials. The structure and morphology of the 13-93 BG powder are shown in Fig. 2. The FT-IR spectrum (Fig. 2a) showed distinctive absorption bands at $490-500 \mathrm{~cm}^{-1}$ (symmetric stretching, $\nu_{\text {sym }}(\mathrm{Si}-\mathrm{O}-\mathrm{Si})$ ), 1000-1300 $\mathrm{cm}^{-1}$ (asymmetric stretching, $\nu_{\text {asym }}(\mathrm{Si}-\mathrm{O}-\mathrm{Si})$ ), $750 \mathrm{~cm}^{-1}$ (bending mode $\delta(\mathrm{Si}-\mathrm{O}-\mathrm{Si}))$ and $930 \mathrm{~cm}^{-1}\left(\mathrm{SiO}_{\mathrm{NBO}}\right.$ shoulder $)$. The XRD pattern of the as-prepared glass (Fig. $2 \mathrm{~b}$ ) showed a band centered at $\sim 30^{\circ}$ in the $2 \theta$ range that was typical of amorphous glass. Fig. $2 \mathrm{c}$ shows the morphology of 13-93 BG powder, which had particle sizes of less than $40 \mu \mathrm{m}$, which facilitated extrusion in 3D printing.

\section{Fabrication and characterization of 3D printed 13-93 BG/SA scaffolds}

Highly concentrated SA, with or without incorporating 13-93 $\mathrm{BG}$, was used to prepare printing pastes to fabricate scaffolds, as shown in Fig. 1. The prepared 13-93 BG/SA composites had mass ratios of $0: 4$ (0\% 13-93 BG), $1: 4$ (20\%), $2: 4$ (33.3\%) and $4: 4(50 \%)$, and the obtained scaffolds were denoted as pure SA, BG/SA $1: 4$, BG/SA $2: 4$ and BG/SA $4: 4$, respectively. All scaffolds were constructed in air and cured by incubation in $\mathrm{CaCl}_{2}$ solution for $5 \mathrm{~h}$ at room temperature. The structures and morphologies of the 3D printed 13-93 BG/SA scaffolds are presented in Fig. 3 and their parameters summarized in Table 1. Top-view and side-view images (Fig. 3a) showed that all printed 13-93 BG/SA scaffolds were completely interconnected and that macropore sizes were better maintained by higher BG contents. High magnification SEM images of the BG/SA 2:4 scaffold (Fig. 3b) not only confirmed a macropore size of around $500 \mu \mathrm{m}$, but also revealed that there were plenty of micropores in the scaffold strands, due to the dissolution of incorporated PVA during scaffold incubation in $\mathrm{CaCl}_{2}$ solution. Moreover, the porosity of 13-93 BG/SA scaffolds (Fig. 3c) was improved from $65.5 \pm 5.3$ to $87.5 \pm 3.9 \%$ as $13-93$ BG content in the scaffolds increased from $0: 4$ to $4: 4$. Fig. 3d depicts the shrinkage ratios in three directions $(X, Y$ and $Z)$ of all 13-93 BG/SA scaffolds. After lyophilization, the pure SA scaffolds demonstrated the most shrinkage, around $20 \%$ in all directions, while the shrinkage ratios decreased markedly to around $10 \%$ (BG/SA $4: 4)$ as the 13-93 BG content increased.

\section{Mechanical properties of 3D printed 13-93 BG/SA scaffolds}

The compressive strength and modulus of the 3D printed 13-93 BG/SA scaffolds in a dry state were determined, as shown in Fig. 4, and used to calculate $70 \%$ deformation. The compressive strength and modulus initially increased, and then decreased, with increasing 13-93 BG content. Pure SA scaffolds had the weakest mechanical properties, with a compressive strength of $9.08 \pm 0.58 \mathrm{MPa}$ and modulus of $42.62 \pm 4.05 \mathrm{MPa}$, while BG/SA $2: 4$ had the most impressive mechanical properties, with a compressive strength of $16.74 \pm 1.78 \mathrm{MPa}$ and modulus of $79.49 \pm 7.38 \mathrm{MPa}$. However, in BG/SA 4:4 scaffolds the compressive strength and modulus sharply declined to $10.94 \pm$ 1.46 MPa and 53.07 $\pm 3.33 \mathrm{MPa}$, respectively.

\section{In vitro mineralization and ionic release of the $3 \mathrm{D}$ printed 13-93 BG/SA scaffolds}

The in vitro apatite mineralization of an implanted material is usually related to its osteoinductive ability for bone regeneration. ${ }^{9,29}$ In this study, the pure SA scaffolds had smooth surfaces without apatite deposition before or after soaking for 10 days in SBF, while incorporation of 13-93 BG endowed the 13-93 BG/SA
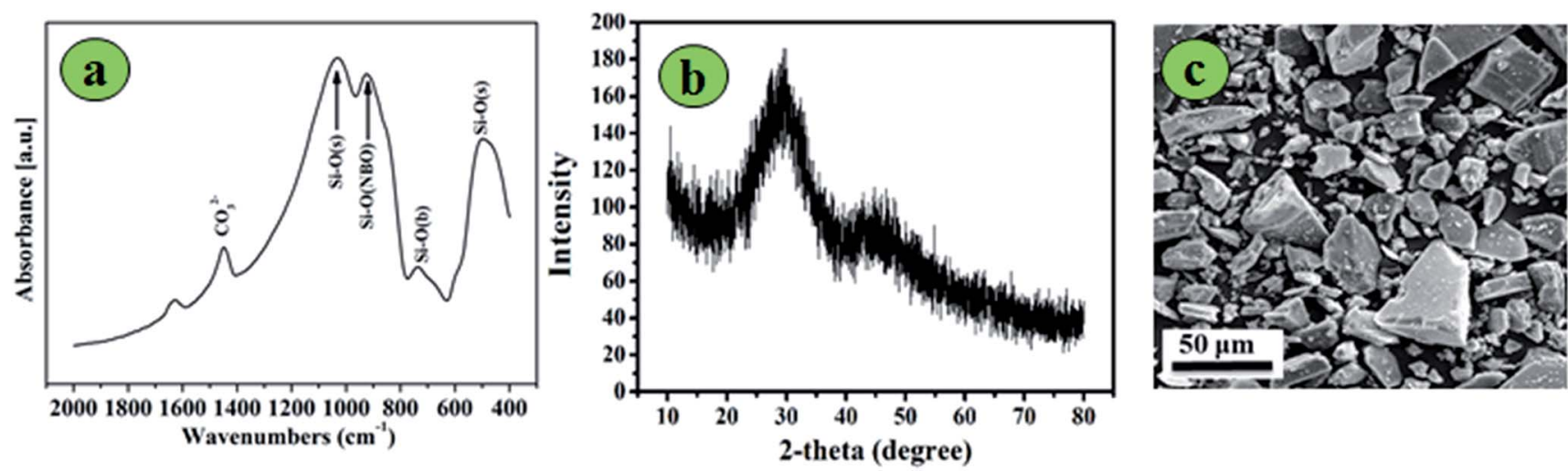

Fig. 2 Characterization of 13-93 BG powders: (a) FT-IR spectrum; (b) XRD pattern; and (c) morphology and size ( $<40 \mu \mathrm{m})$. 


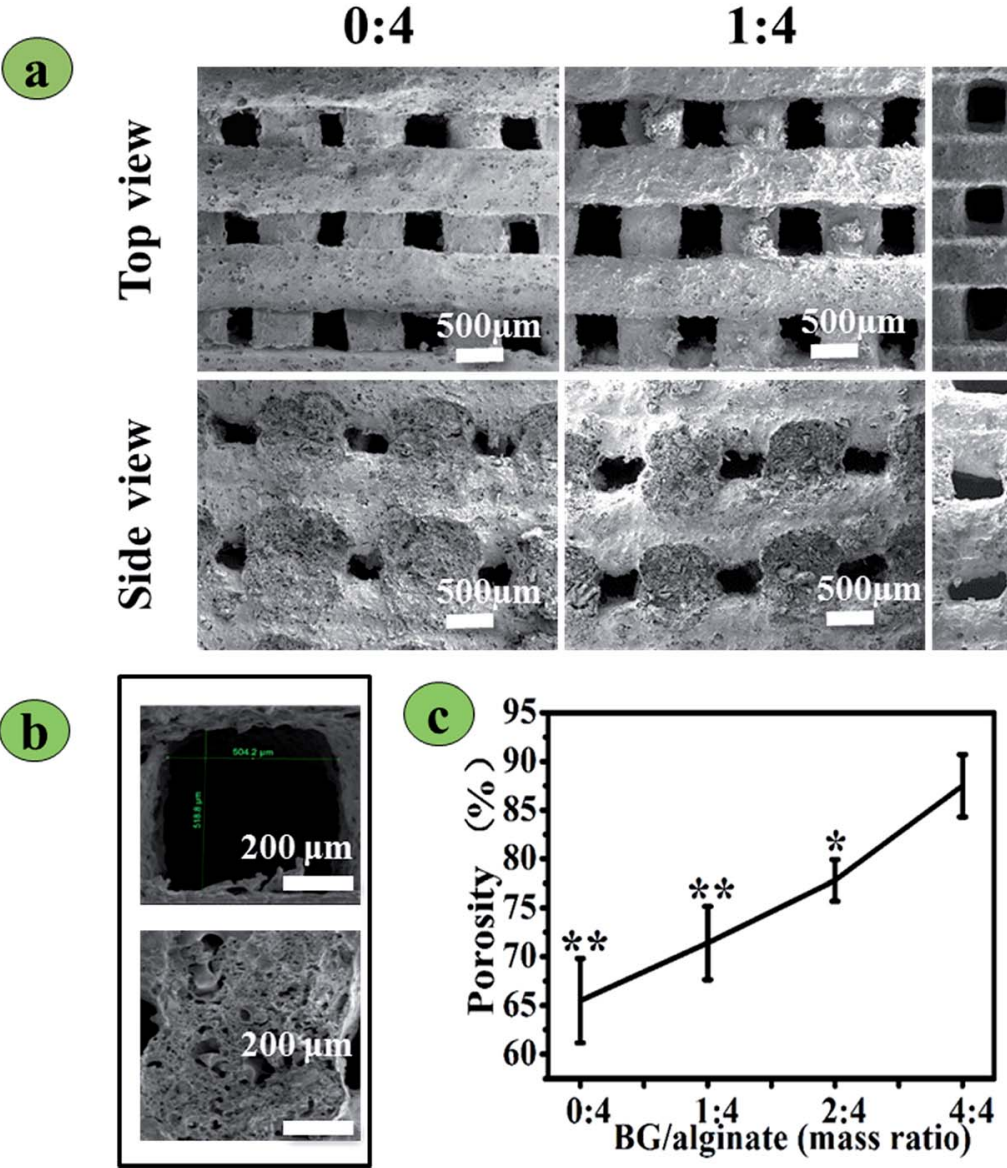

$2: 4$
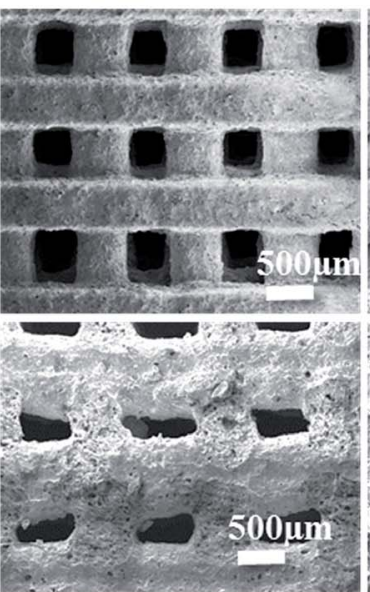

$4: 4$

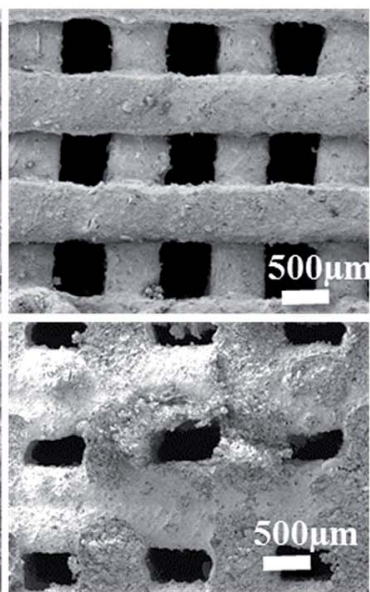

Fig. 3 Morphology, porosity and shrinkage of 3D-printed BG/SA composite scaffolds: (a) top and side-view SEM images of scaffolds. BG/SA mass ratios were $0: 4,1: 4,2: 4$, and $4: 4 ;$ (b) high-magnification SEM images showing a pore and a vertical section of strands of the BG/SA $2: 4$ scaffold; (c) porosity; (d) scaffold shrinkage (\%) in $X, Y$ and $Z$ directions. Scale bar is $500 \mu \mathrm{m}$. (*Denotes $P<0.05$, compared with 13-93 BG/SA $0: 4$; **denotes $P<0.01$, compared with 13-93 BG/SA $0: 4)$.

Table 1 Parameters of 3D printed BG/SA composite scaffolds

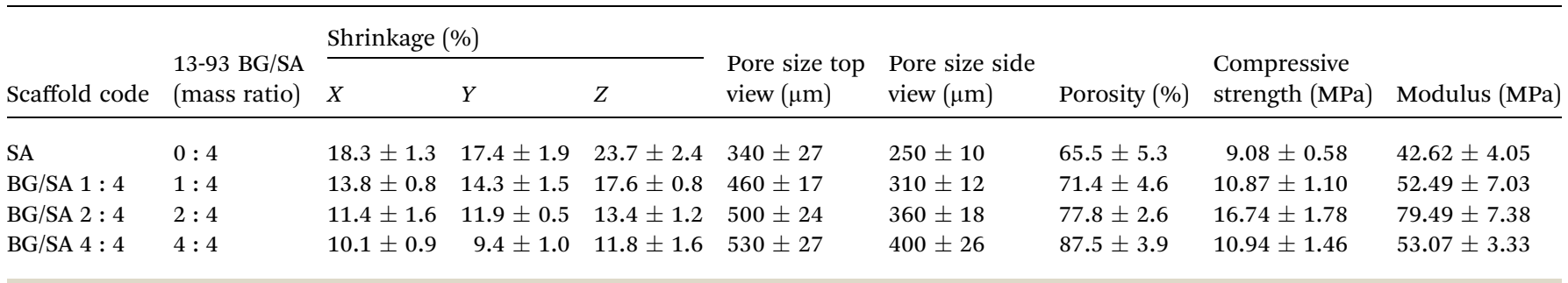

Table 2 Primer pairs used in real-time PCR analysis

\begin{tabular}{llll}
\hline Gene & Forward primer $\left(5^{\prime}-3^{\prime}\right)$ & Reverse primer $\left(5^{\prime}-3^{\prime}\right)$ & Accession no. \\
\hline GAPDH & GGCACAGTCAAGGCTGAGAATG & ATGGTGGTGAAGACGCCAGTA & NM_017008.4 \\
OPN & CCAAGCGTGGAAACACACAGCC & GGCTTTGGAACTCGCCTGACTG & M14656.1 \\
OCN & GGTGCAGACCTAGCAGACACCA & AGGTAGCGCCGGAGTCTATTCA & J04500 \\
COL-I & GCCTCCCAGAACATCACCTA & GCAGGGACTTCTTGAGGTTG & NM_053304 \\
ALP & AACGTGGCCAAGAACATCATCA & TGTCCATCTCCAGCCGTGTC & NM_013059 \\
\hline
\end{tabular}

scaffolds with excellent apatite mineralization ability in SBF (Fig. 5a). After soaking for 10 days in PBS, alveolate-like apatite crystals formed on the surface of BG/SA $1: 4$, BG/SA $2: 4$ and
BG/SA $4: 4$. Newly formed apatite on the surface of the 13-93 BG/SA scaffolds was further confirmed by EDS analysis, as shown in Fig. 5b, with characteristic peaks observed for $\mathrm{Ca}, \mathrm{Mg}$, 

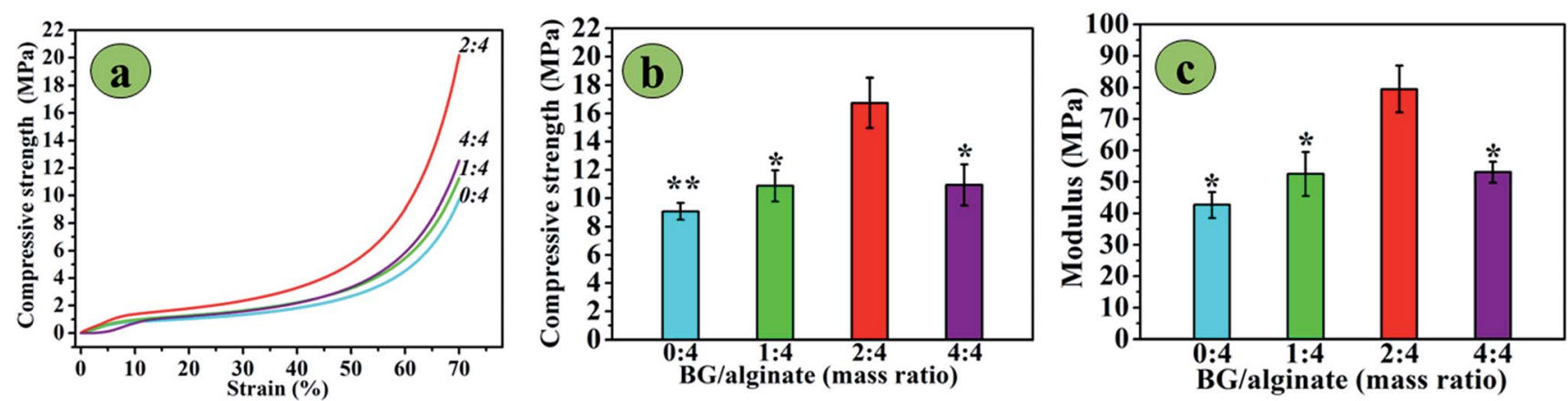

Fig. 4 Mechanical properties of 3D-printed BG/SA composite scaffolds: (a) compressive stress-strain curves; (b) compressive strength; and (c) modulus. (*Denotes $P<0.05$ compared with BG/SA $2: 4$; **denotes $P<0.01$ compared with BG/SA $2: 4, n=4$ ).

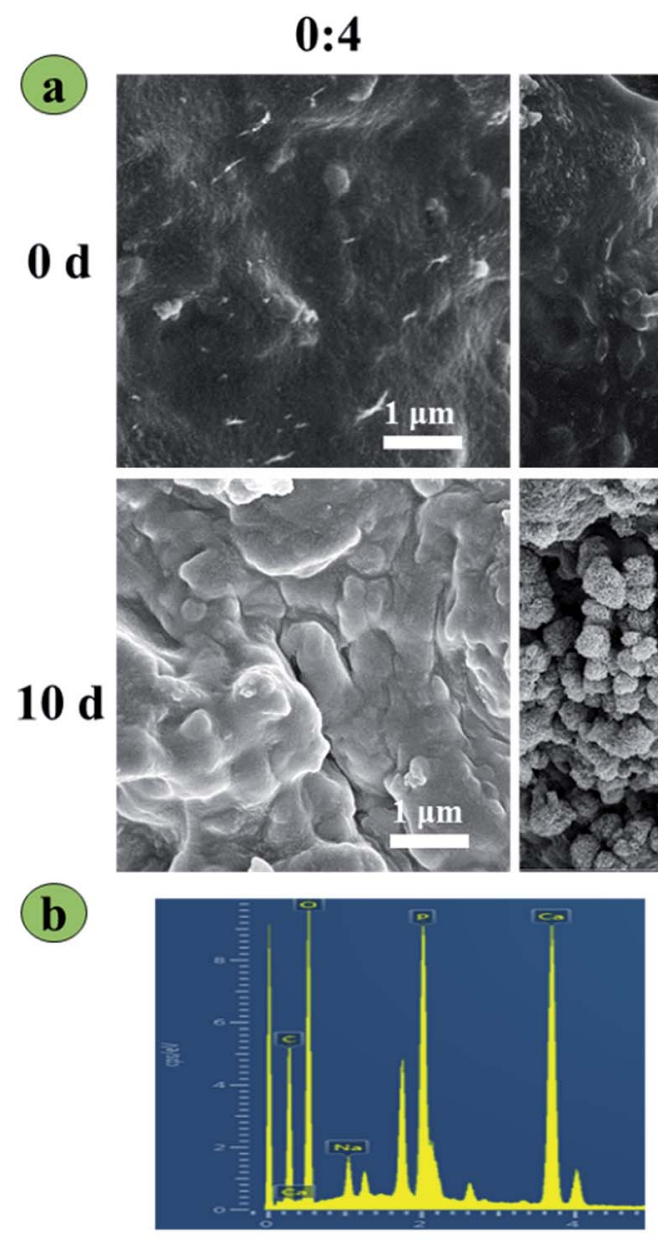

$1: 4(\mathrm{Ca} / \mathrm{P}=1.37)$

$1: 4$
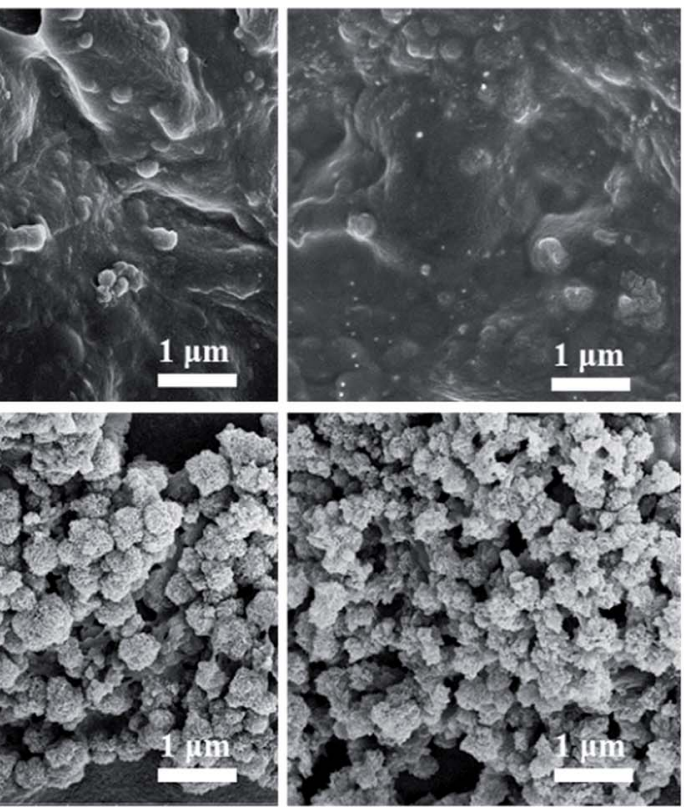

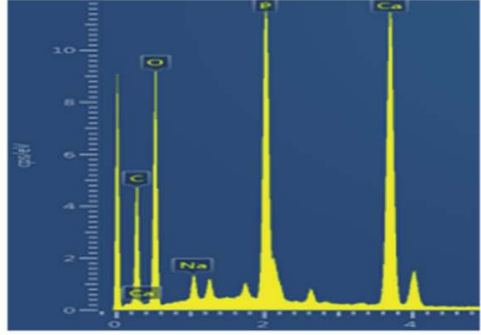

$2: 4(\mathrm{Ca} / \mathrm{P}=1.34)$

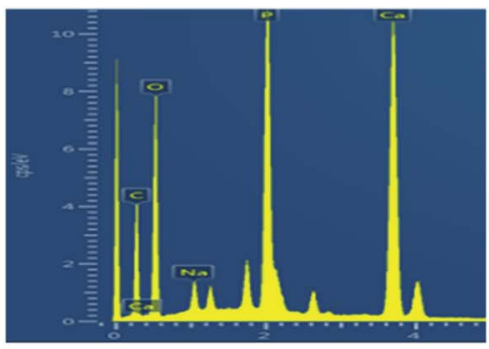

$4: 4(\mathrm{Ca} / \mathrm{P}=1.33)$

Fig. 5 In vitro apatite mineralization of 3D-printed BG/SA composite scaffolds: (a) surface morphologies of scaffolds before and after soaking in SBF for 10 days; (b) EDS analyses of apatite layers. Scale bar is $1 \mu \mathrm{m}$.

$\mathrm{Si}$ and $\mathrm{P}$. Moreover, the $\mathrm{Ca} / \mathrm{P}$ ratios in $\mathrm{BG} / \mathrm{SA} 1: 4, \mathrm{BG} / \mathrm{SA} 2: 4$ and BG/SA $4: 4$ were $1.37,1.34$ and 1.33 , respectively. All Ca/P ratios were less than that of hydroxyapatite (1.67), which might be attributed to the presence of $\mathrm{Mg}^{2+}$ replacing $\mathrm{Ca}^{2+}$ in the mineralized apatite layer.

The $\mathrm{pH}$ values of PBS solutions containing four types of scaffolds were measured over 7 days, as shown in Fig. 6a. A pH variation of solutions containing BG/SA scaffold rapidly increased over 7 days, while the $\mathrm{pH}$ of that containing pure SA scaffold increased slowly. After immersing the scaffolds for 3 days, the $\mathrm{pH}$ values of each BG/SA scaffold were higher than that of the pure SA scaffold, with the $\mathrm{pH}$ value higher with increasing 13-93 BG content. In BG/SA $4: 4$, the $\mathrm{pH}$ value was greater than 8.5 after immersion for 7 days in PBS. Meanwhile, the 
(a)

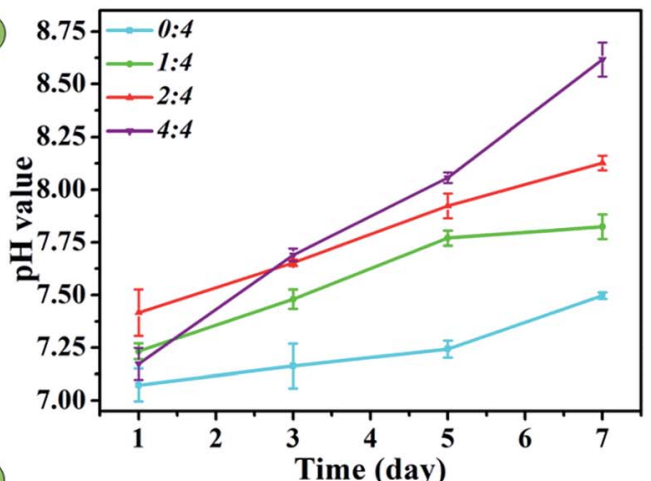

c)

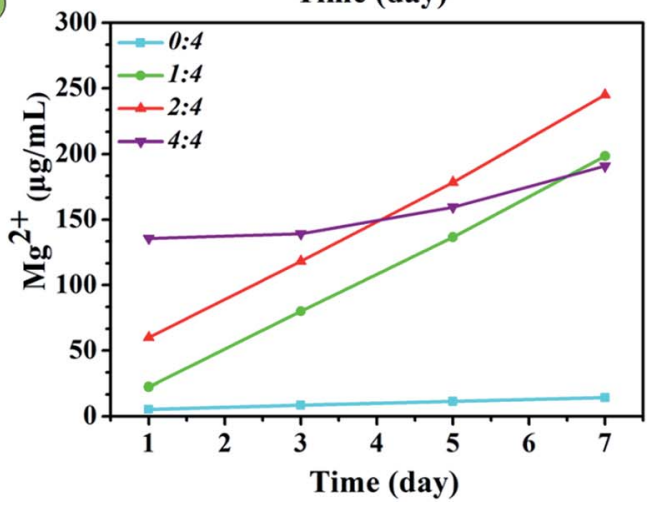

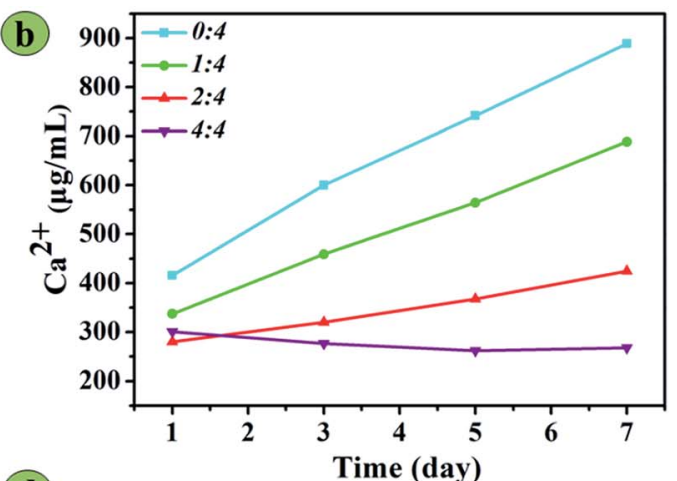

d

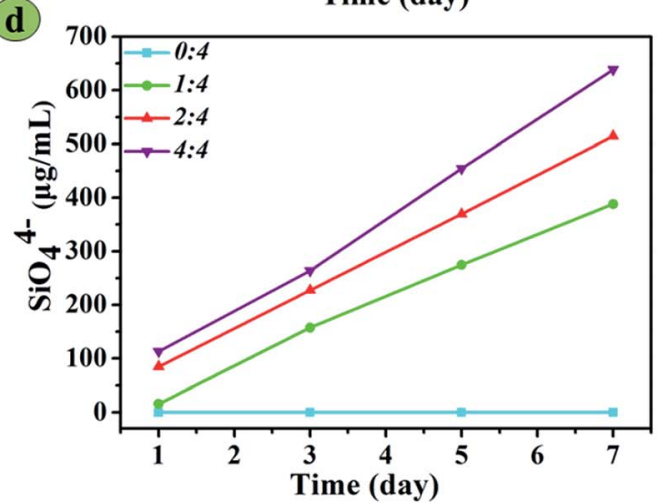

Fig. 6 pH value and ion release of 3D-printed BG/SA composite scaffolds in PBS solution at 1, 3, 5 and 7 days: (a) pH variation; (b) $\mathrm{Ca}^{2+}$ release; (c) $\mathrm{Mg}^{2+}$ release; and (d) $\mathrm{SiO}_{4}^{4-}$ release.

(a)

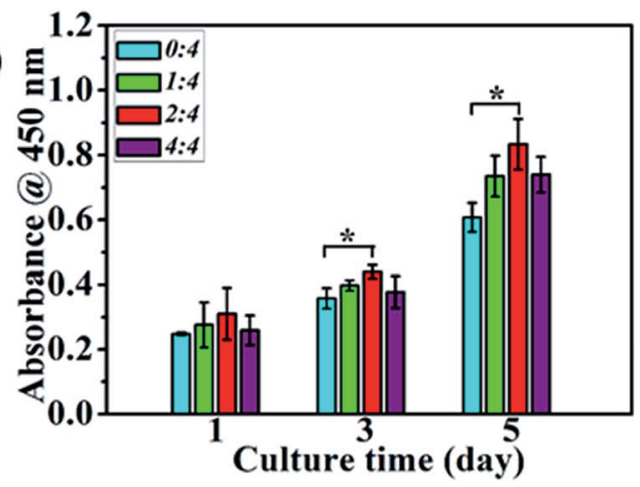

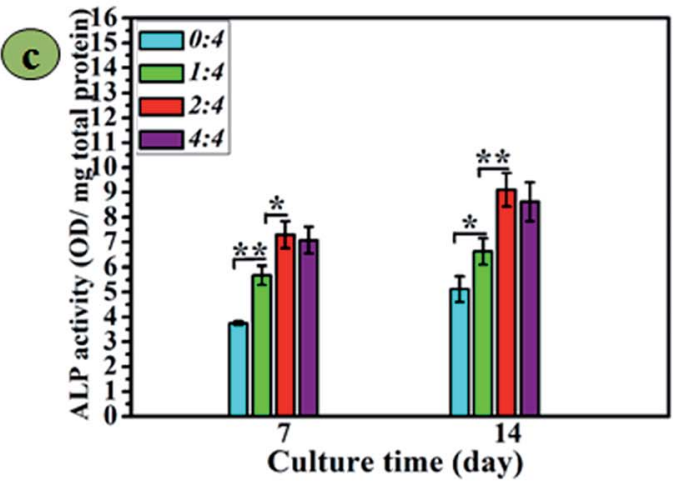

(b)
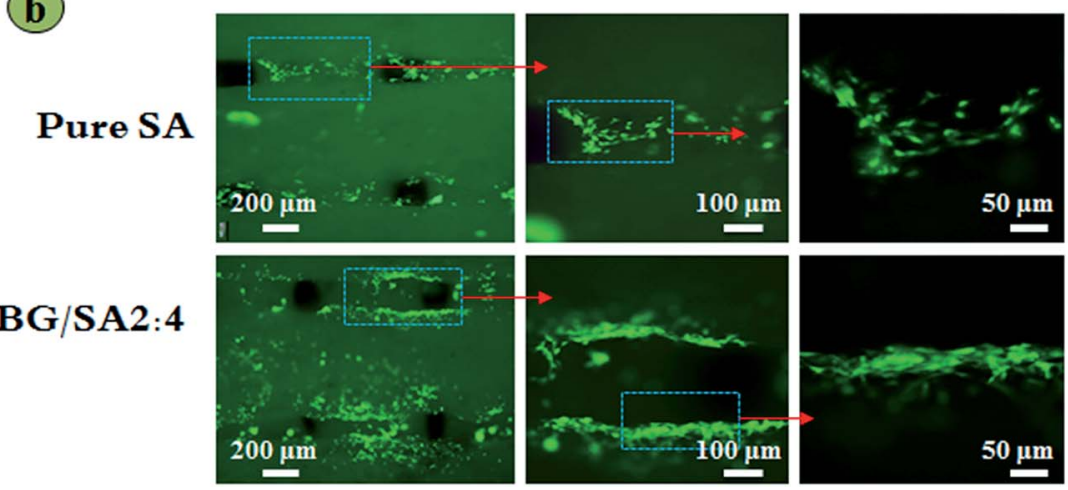

Fig. 7 rBMSC response on 3D-printed BG/SA composite scaffolds: (a) proliferation determined using CCK- 8 at 1, 3 and 5 days; (b) attachment on pure SA and BG/SA 2 : 4 scaffolds determined by fluorescence images of cells after $36 \mathrm{~h}$ of culture; and (c) osteogenic differentiation of rBMSC seeded on scaffolds at 7 and 14 days. ( $n=3$, mean $\pm S D$, *denotes $p<0.05, * *$ denotes $p<0.01$ ). 


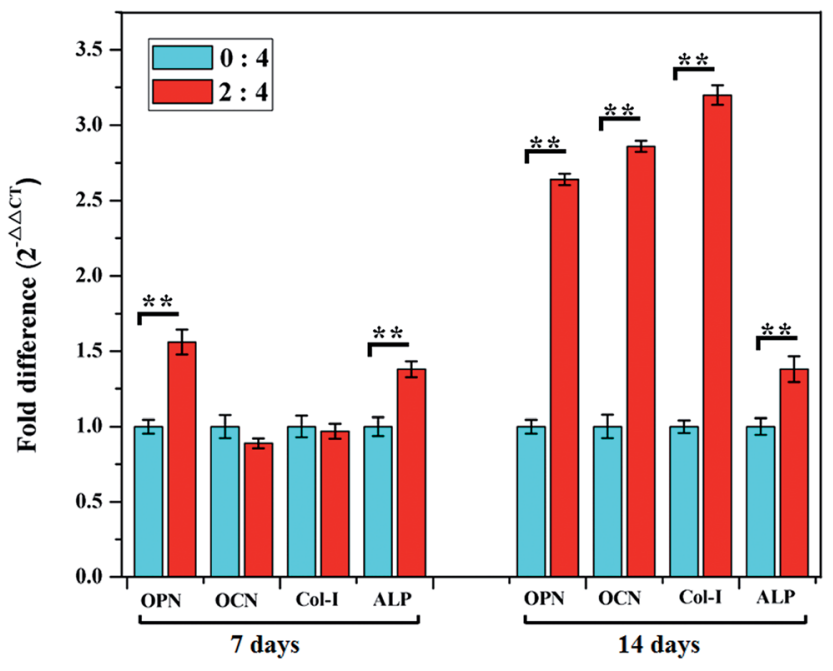

Fig. 8 Osteogenic gene expression (OPN, OCN, Col-I and ALP) of rBMSCs cultured on pure SA and BG/SA $2: 4$ scaffolds at 7 and 14 days of culture. (*Denotes $p<0.05$, **denotes $p<0.01$ ).

concentrations of $\mathrm{Ca}^{2+}, \mathrm{Mg}^{2+}$ and $\mathrm{SiO}_{4}{ }^{4-}$ ions in PBS after soaking the scaffolds were detected by ICP over 7 days. Fig. $6 \mathrm{~b}$ shows that the concentration of $\mathrm{Ca}^{2+}$ ions from pure SA, BG/SA $1: 4$ and BG/SA $2: 4$ in PBS increased, but the concentration of $\mathrm{Ca}^{2+}$ ions from BG/SA $4: 4$ in PBS slowly declined. The $\mathrm{Ca}^{2+}$ concentration could be increased by ion exchange from the alginate strands and the release of 13-93 BG, but decreased by apatite mineralization. Therefore, $\mathrm{Ca}^{2+}$ release from the alginate strands motivated the sharp increase in $\mathrm{Ca}^{2+}$ ions from the pure SA scaffold in PBS. In contrast, BG/SA $4: 4$, the scaffold with the highest BG content, gave the highest $\mathrm{pH}$ value in PBS, which stimulated the spontaneous formation of apatite mineralization, causing a sharp decrease in $\mathrm{Ca}^{2+}$ concentration. The release of $\mathrm{Mg}^{2+}$ and $\mathrm{SiO}_{4}{ }^{4-}$ ions (Fig. $6 \mathrm{c}$ and d) was attributed to 13-93 BG incorporation, resulting in improved $\mathrm{Mg}^{2+}$ and $\mathrm{SiO}_{4}{ }^{4-}$ concentrations with increasing 13-93 BG content, except for $\mathrm{Mg}^{2+}$ release from BG/SA $4: 4$. This exception was due to $\mathrm{Mg}^{2+}$ ions, in a similar fashion to $\mathrm{Ca}^{2+}$ ions, precipitating under the highly alkaline conditions provided by BG/SA $4: 4$.

\section{Cultivation of rBMSCs on 3D printed 13-93 BG/SA scaffolds}

To evaluate the biological effects of the 3D printed 13-93 BG/SA scaffolds for bone regeneration, the cell morphology, proliferation, ALP activity and osteogenic activity of rBMSCs seeding on the scaffolds were systematically investigated. The proliferation of rBMSCs on different scaffolds is depicted in Fig. 7a. The cell number increased on all scaffolds during the 5 day culture, and rBMSCs on BG/SA scaffolds proliferated better than those on pure SA scaffolds. However, when the BG content was increased to $50 \%(4: 4)$, the proliferation of rBMSCs declined. This was further confirmed by fluorescence images (Fig. 7b), which showed that rBMSCs preferred to spread and adhere on BG/SA $2: 4$ scaffolds than pure SA scaffolds.

Determination of the ALP activity of rBMSCs (Fig. 7c) indicated that 13-93 BG incorporation stimulated osteogenic differentiation of rBMSC. After 7 and 14 days of culture, the ALP activity of rBMSCs cultured on BG/SA composite scaffolds was significantly higher than that on pure alginate scaffolds $(p<$ 0.05). However, the ALP activities of rBMSCs on BG/SA $4: 4$, in accordance with the proliferation results, were slightly weaker than those on BG/SA $2: 4$ scaffolds, but better than those on pure SA scaffolds.

Fig. 8 summarizes the osteogenesis-related gene expressions of OPN, OCN, Co1-I and ALP at 7 and 14 days of incubation with rBMSCs on BG/SA 2: 4 composite and pure SA scaffolds. At 7 days, OPN and ALP of rBMSCs showed higher expression on BG/ SA 2 : 4 scaffolds than on pure SA scaffolds, while OCN and Col-I expression on pure SA scaffolds and BG/SA $2: 4$ scaffolds were similar. Furthermore, at 14 days, the expression of OPN, OCN, Col-I and ALP on BG/SA $2: 4$ scaffolds were significantly higher than those on pure SA scaffolds. These results were likely due to incorporation of 13-93 BG onto BG/SA scaffolds, which could promote osteogenic differentiation of rBMSCs.

\section{Discussion}

In the present study, biodegradable 13-93 BG/SA composite scaffolds were successfully fabricated by 3D printing under mild conditions with an XXYY pattern. Using SA components as the organic matrix, gelatinized by crosslinking with $\mathrm{Ca}^{2+}$ to form the steady scaffolds, is a popular stabilization method for mild 3D printing, while 13-93 BG particles with diameters less than 40 $\mu \mathrm{m}$ were used as the inorganic component (Fig. 2), with the intention of improving the mechanical properties and bioactivity of the SA scaffold. Compared with previous methods, ${ }^{26-29}$ including slip casting, polymer foam replication and freeze extrusion, this was a more facile method for preparing 13-93 BG scaffolds for bone regeneration. Meanwhile, the incorporation of 13-93 BG into SA scaffolds significantly improved the mechanical strength, apatite mineralization ability, and attachment and osteogenic differentiation of rBMSC.

Bioactive scaffolds with interconnected pores, suitable porosity and tailored mechanical strength are needed for bone tissue engineering. ${ }^{30,34}$ In particular, the 13-93 BG/SA composite scaffolds obtained herein were observed to have hierarchical macropores (around $500 \mu \mathrm{m}$, Fig. 3a) and plenty of micropores on the strands (Fig. 3b), suggesting completely interconnected pores, which have been reported as suitable for bone-related cell ingrowth and nutrient delivery. ${ }^{34}$ Meanwhile, increasing 13-93 BG content improved porosity and decreased shrinkage ratios in three directions in the 3D printed 13-93 BG/SA composite scaffolds (Fig. $3 \mathrm{c}$ and d), further indicating that 1393 BG dispersed in the SA matrix helped maintain composite scaffold stability. Furthermore, the 13-93 BG content was found to significantly regulate the mechanical properties of the 13-93 BG/SA composite scaffolds (Fig. 4), giving a maximum compressive strength and modulus of $16.74 \pm 1.78 \mathrm{MPa}$ and $79.49 \pm 7.38 \mathrm{MPa}$, respectively, in 13-93 BG/SA $2: 4$. On one hand, the 13-93 BG particles acted as reinforcing agents in composites when the 13-93 BG content increased from 0 to $33.3 \%$. On the other hand, when the 13-93 BG content became more dominant in the composite scaffolds, as in BG/SA $4: 4$, 
they exhibited the brittleness of 13-93 BG. Accordingly, 13-93 BG/SA composite scaffolds with porosities of $65-87 \%$, compressive strengths of 10-16 MPa and moduli of 40-80 Mpa met the mechanical demands of human cancellous bone. ${ }^{29}$

In addition to sufficient mechanical properties, excellent cytocompatibility and sound bioactivity are also indispensable. In vitro apatite mineralization of an implanted material in SBF is known as the initial evaluation of osteoinductive ability for bone regeneration.,33,34 Our study showed that 13-93 BG incorporation significantly improved the apatite forming ability of SA scaffolds in SBF (Fig. 5). The improved apatitemineralization ability of SA scaffolds might benefit the rBMSC response to scaffolds because improved cell attachment and higher specific ALP activity were observed for 13-93 BG/SA compared with pure SA scaffolds. 13-93 BG incorporation also led to the release of $\mathrm{Mg}^{2+}$ and $\mathrm{SiO}_{4}{ }^{4-}$ ions (Fig. 6), which were generally recognized as enhancers of bone regeneration. Previous investigations have confirmed that $\mathrm{Mg}^{2+}$ and $\mathrm{SiO}_{4}{ }^{4-}$ ions not only improve cell attachment and proliferation, but also simulate osteogenic differentiation of rBMSCs. $^{35,36}$ In addition, environment $\mathrm{pH}$ also plays an important role in cell growth, proliferation and mineralization. For bone regeneration, it has been reported that a relatively high local $\mathrm{pH}$ is necessary during the bone formation process because the optimum ALP occurs at $\mathrm{pH}$ 8.5, in comparison with the nominal physiological value of 7.4. ${ }^{37}$ Furthermore, 13-93 BG was also shown to degrade the release of alkaline ions, which would exchange with hydrogen ions $\left(\mathrm{H}^{+}\right)$in water to generate a weakly alkaline $\mathrm{pH}$. This $\mathrm{pH}$ would promote the nucleation of calcium phosphates and facilitate the proliferation of osteoblasts. The results of this study showed that 13-93 BG incorporation into scaffolds immersed in PBS increased the environment $\mathrm{pH}$ (Fig. 6). Therefore, the rBMSC activities, comprising attachment (Fig. 7), proliferation (Fig. 7) and mineralization (Fig. 8), on 1393 BG/SA 2:4 were the highest, and were simultaneously regulated by three factors, namely apatite mineralization, the release of bioactive $\mathrm{Mg}^{2+}$ and $\mathrm{SiO}_{4}{ }^{4-}$ ions, and the environment $\mathrm{pH}$.

\section{Conclusions}

In this study, we fully utilized the crosslinking property of SA to fabricate 13-93 BG/SA scaffolds by 3D printing under mild conditions. All the obtained scaffolds were completely interconnected, with pore sizes, porosities and shrinkage ratios that were regulated by the amount of 13-93 BG incorporated. Moreover, in comparison with the pure SA scaffold, 13-93 BG incorporation significantly improved the mechanical properties, increased apatite mineralization and released bioactive $\mathrm{Mg}^{2+}$ and $\mathrm{SiO}_{4}{ }^{4-}$ ions. Meanwhile, rBMSC preferred to spread, adhere and osteogenically differentiate on the 13-93 BG/SA scaffolds, especially BG/SA $2: 4$. Therefore, the 3D printed 1393 BG/SA scaffolds, a novel biodegradable organic-inorganic composite scaffold with suitable porosity, tailored mechanical strength and sound bioactivity, could be a potential candidate for bone regeneration.

\section{Acknowledgements}

The authors gratefully acknowledge financial support from the Science and Technology Projects of Shenzhen (JCYJ20140417113430596, CXZZ20140417 113430716), the Science and Technology project of Guangdong Province (2015A010105021) and the Youth Talents of Guangdong Science and Technology Innovation (2015TQ01X076), and the Shenzhen Peacock Innovation Team (No. 110811003586331).

\section{References}

1 M. Xu, D. Zhai, J. Chang and C. Wu, Acta Biomater., 2014, 10, 463-476.

2 D. W. Hutmacher, Biomaterials, 2000, 21, 2529-2543.

3 Y. Luo, A. Lode and M. Gelinsky, Adv. Healthcare Mater., 2013, 2, 777-783.

4 Y. Luo, C. Wu, A. Lode and M. Gelinsky, Biofabrication, 2012, 5, 015005.

5 U. Kalsoom, P. N. Nesterenko and B. Paull, RSC Adv., 2016, 6, 60355-60371.

6 Y. Yu, S. Hua, M. Yang, Z. Fu, S. Teng, K. Niu, Q. Zhao and C. Yi, RSC Adv., 2016, 6, 110557-110565.

7 S. S. Kim, K. M. Ahn, M. S. Park, J. H. Lee, C. Y. Choi and B. S. Kim, J. Biomed. Mater. Res., Part A, 2007, 80, 206-215.

8 A. K. Miri, N. Muja, N. O. Kamranpour, W. C. Lepry, A. R. Boccaccini, S. A. Clarke and S. N. Nazhat, Biomaterials, 2016, 85, 128-141.

9 L. L. Hench and J. R. Jones, Front. Bioeng. Biotechnol., 2015, 3, 194.

10 C. Wu, J. Chang, W. Zhai and S. Ni,J. Mater. Sci.: Mater. Med., 2007, 18, 857-864.

11 Q. Z. Chen, I. D. Thompson and A. R. Boccaccini, Biomaterials, 2006, 27, 2414-2425.

12 F. Zhao, W. Zhang, X. Fu, W. Xie and X. Chen, RSC Adv., 2016, 6, 91201-91208.

13 P. X. Ma, Mater. Today, 2004, 7, 30-40.

14 E. Nejati, H. Mirzadeh and M. Zandi, Composites, Part A, 2008, 39, 1589-1596.

15 M. Okamoto and B. John, Prog. Polym. Sci., 2013, 38, 14871503.

16 J. Russias, E. Saiz, S. Deville, K. Gryn, G. Liu, R. K. Nalla and A. P. Tomsia, J. Biomed. Mater. Res., Part A, 2007, 83A, 434445.

17 C. Wu, Y. Luo, G. Cuniberti, Y. Xiao and M. Gelinsky, Acta Biomater., 2011, 7, 2644-2650.

18 A. Lode, K. Meissner, Y. Luo, F. Sonntag, S. Glorius, B. Nies, C. Vater, F. Despang, T. Hanke and M. Gelinsky, J. Tissue Eng. Regen. Med., 2014, 8, 682-693.

19 G. Jin and G. H. Kim, J. Mater. Chem., 2011, 21, 17710-17718. 20 Y. Luo, A. Lode, C. Wu, J. Chang and M. Gelinsky, ACS Appl. Mater. Interfaces, 2015, 7, 6541-6549.

21 G. Luo, J. Wang, Y. Wang, B. Feng and J. Weng, J. Microencapsulation, 2015, 32, 129-136.

22 K. Y. Lee and D. J. Mooney, Prog. Polym. Sci., 2012, 37, 106126. 
23 X.-F. Wang, P.-J. Lu, Y. Song, Y.-C. Sun, Y.-G. Wang and Y. Wang, RSC Adv., 2016, 6, 6832-6842.

24 Y. Luo, G. Luo, M. Gelinsky, P. Huang and C. Ruan, Mater. Lett., 2017, 189, 295-298.

25 A. Hoppe, B. Sarker, R. Detsch, N. Hild, D. Mohn, W. Stark and A. Boccaccini, J. Non-Cryst. Solids, 2014, 387, 41-46.

26 A. M. Deliormanl, Mater. Sci. Eng. C, 2015, 53, 262-271.

27 Q. Fu, M. N. Rahaman, B. S. Bal and R. F. Brown, J. Biomed. Mater. Res., Part A, 2010, 93A, 1380-1390.

28 X. Liu, M. N. Rahaman, G. E. Hilmas and B. S. Bal, Acta Biomater., 2013, 9, 7025-7034.

29 Q. Fu, E. Saiz, M. N. Rahaman and A. P. Tomsia, Mater. Sci. Eng., C, 2011, 31, 1245-1256.

30 H. R. Ramay and M. Zhang, Biomaterials, 2003, 24, 3293-3302.

31 C. Maniatopoulos, J. Sodek and A. H. Melcher, Cell Tissue Res., 1988, 254, 317-330.
32 E. Y. Lee, Y. Xia, W. S. Kim, M. H. Kim, T. H. Kim, K. J. Kim, B. S. Park and J. H. Sung, Wound Repair Regen, 2009, 17, 540547.

33 H. Mori, T. Fujitani, Y. Kanemura, M. Kino-Oka and M. Taya, J. Biosci. Bioeng., 2007, 104, 231-234.

34 H. J. Park, O. J. Lee, M. C. Lee, B. M. Moon, H. W. Ju, J. Min Lee, J.-H. Kim, D. W. Kim and C. H. Park, Int. J. Biol. Macromol., 2015, 78, 215-223.

35 C. Liu, X. Fu, H. Pan, P. Wan, L. Wang, L. Tan, K. Wang, Y. Zhao, K. Yang and P. K. Chu, Sci. Rep., 2016, 6, 27374.

36 J. S. Fernandes, M. Martins, N. M. Neves, M. H. Fernandes, R. L. Reis and R. A. Pires, ACS Biomater. Sci. Eng., 2016, 2, 1143-1150.

37 W. Liu, T. Wang, C. Yang, B. W. Darvell, J. Wu, K. Lin, J. Chang, H. Pan and W. W. Lu, Osteoporosis Int., 2016, 27, 93-104. 